\title{
ON THE FACIAL STRUCTURE OF THE UNIT BALLS IN A JBW*-TRIPLE AND ITS PREDUAL
}

\author{
C. M. EDWARDS AND G. T. RÜTTIMANN
}

\begin{abstract}
The set of tripotents in a $\mathrm{JBW}^{*}$-triple $\mathfrak{U}$ with its natural ordering and with a largest element adjoined is shown to be a complete lattice, order isomorphic to the lattice of norm closed faces in the unit ball $\mathfrak{U}_{* 1}$ of the predual $\mathfrak{U}_{*}$ of $\mathfrak{U}$ and anti-order isomorphic to the lattice of weak closed faces of the unit ball $\mathfrak{U}_{1}$ in $\mathfrak{U}$. As a consequence, the set of partial isometries in a $\mathbf{W}^{*}$-algebra with its natural ordering and again with a largest element adjoined forms a complete lattice and every non-empty weak* closed face of its unit ball is of the form

$$
u+\left(1-u u^{*}\right) \mathfrak{U}\left(1-u^{*} u\right)_{1}
$$
\end{abstract}

for some unique partial isometry $u$.

\section{Introduction}

In previous papers $[9,10,11]$ the authors explored the facial structure of the closed unit balls in JBW-algebras and their preduals, and in JB-algebras and their duals. It was shown that the faces, closed in the relevant topology, can be described by means of elements of the complete lattice of idempotents in the first case in the JBW-algebra itself and in the second case in the enveloping JBW-algebra. Most of the techniques used in those papers do not lend themselves to the study of the facial structure of unit balls in complex Banach spaces, in particular the complexifications of JB-algebras and JBW-algebras known respectively as Jordan $\mathrm{C}^{*}$-algebras [33] (or $\mathrm{JB}^{*}$-algebras [34]) and Jordan $\mathrm{W}^{*}$-algebras [8] (or JBW*-algebras [34]). The sets of such algebras respectively include the sets of $C^{*}$-algebras and $W^{*}$-algebras. The open unit balls in Jordan $\mathrm{C}^{*}$-algebras are bounded symmetric domains which generalise the classical bounded symmetric domains in finite-dimensional complex vector spaces $[24,27]$. However, the most general bounded symmetric domains in infinitedimensional complex Banach spaces are isomorphic to the open unit balls, not in Jordan $\mathrm{C}^{*}$-algebras, but in the wider class of $\mathrm{JB}^{*}$-triples. Therefore, JB*-triples and those $\mathrm{JB}^{*}$-triples which are dual spaces, the $\mathrm{JBW}^{*}$-triples, not only form natural generalisations of Jordan $\mathrm{C}^{*}$-algebras and $\mathrm{C}^{*}$-algebras, and Jordan $\mathrm{W}^{*}$-algebras and $\mathrm{W}^{*}$-algebras, but also provide a context for the study of infinite-dimensional holomorphy and infinite-dimensional Lie algebras [25].

Recently the structure theory of $\mathrm{JB}^{*}$-triples and $\mathrm{JBW}^{*}$-triples has received considerable attention $[4,5,12$ to 18,22$]$. In this paper the facial structure of the unit balls in a $\mathrm{JBW}^{*}$-triple and its predual are studied. One of the methods used in the investigation of real Banach spaces which is available in the complex case is the use of the mappings $E \rightarrow E^{\prime}$ and $F \rightarrow F$, between subsets of the unit balls $V_{1}$ and $V_{1}^{*}$ in a Banach space $V$ and its dual $V^{*}$ defined by

$$
\begin{aligned}
E^{\prime} & =\left\{a: a \in V_{1}^{*}, a(x)=1, \forall x \in E\right\}, \\
F_{1} & =\left\{x: x \in V_{1}, a(x)=1, \forall x \in F\right\} .
\end{aligned}
$$

Received 11 April 1987

1980 Mathematics Subject Classification (1985 Revision) 46B20.

J. London Math. Soc. (2) 38 (1988) 317-332 
The ranges of these mappings are the complete lattices $S_{w^{*}}\left(V_{1}^{*}\right)$ and $S_{n}\left(V_{1}\right)$ of weak ${ }^{*}$-semi-exposed faces of $V_{1}^{*}$ and norm-semi-exposed faces of $V_{1}$ respectively. This method, so effective in $[10,11]$, is also extremely useful in the study of $\mathrm{JBW}^{*}$-triples. In this case the role played by idempotents in the theory of JBW-algebras is taken by elements of the set $\mathscr{U}(\mathfrak{U})$ of tripotents in the $\mathrm{JBW}^{*}$-triple $\mathfrak{A}$. Indeed, the first main result of the paper shows that every norm closed face of the unit ball $\mathfrak{U}_{* 1}$ in the predual $\mathfrak{U}_{*}$ of $\mathfrak{A}$ which is not $\mathfrak{U}_{* 1}$ itself is of the form $\{u\}$, for some unique element $u$ of $\mathscr{U}(\mathfrak{U})$, that with respect to the natural partial ordering $\mathscr{U}(\mathfrak{H})$ with a largest element adjoined forms a complete lattice $\mathscr{U}(\mathfrak{H})^{\mathcal{\nu}}$, and that the mapping $u \rightarrow\{u\}$, is an order isomorphism from $\mathscr{U}(\mathfrak{R})^{\sim}$ onto the complete lattice $\mathscr{F}_{n}\left(\mathfrak{H}_{* 1}\right)$ of norm closed faces of $\mathfrak{U}_{* 1}$. The second main result shows that every non-empty weak* closed face of $\mathfrak{Q}_{1}$ is of the form $\{u\}$, for some element $u$ of $\mathscr{U}(\mathfrak{H})$ and that the mapping $u \rightarrow\{u\}$,' extends to an anti-order isomorphism from $\mathscr{U}(\mathfrak{U})^{\sim}$ onto the complete lattice $\mathscr{F}_{w *}\left(\mathfrak{H}_{1}\right)$ of weak ${ }^{*}$ closed faces of $\mathfrak{U}_{1}$. An important consequence of this result is that the non-empty weak* closed faces of the unit ball in a $W^{*}$-algebra $\mathfrak{U}$ are all of the form $u+\left(1-u u^{*}\right) \mathfrak{X}\left(1-u^{*} u\right)_{1}$, where $u$ is a partial isometry in $\mathfrak{A}$ with initial projection $u^{*} u$ and final projection $u u^{*}$. Neither this result nor the fact that the set of partial isometries in a $\mathrm{W}^{*}$-algebra with a largest element adjoined forms a complete lattice in which the lattice of projections appears as a principal ideal seems to have been previously observed.

The paper is organized as follows. In $\S 2$ various preliminary definitions and results are given. In $\S 3$ the properties of the set $\mathscr{U}(\mathfrak{U})$ of tripotents in $\mathfrak{U}$ and its connection with the local facial structure of $\mathfrak{A}_{1}$ and $\mathfrak{Q}_{*_{1}}$ are described. Since it is the aim of the authors to make the paper reasonably self-contained, whilst most of the results of $\$ 2,3$ are new both sections owe much to [16]. It should be said however that some of the results in [16] were proved under additional assumptions which were later shown to be redundant $[4,5]$. The main results are proved in $\S 4$, and $\S 5$ is devoted to a study of special cases, particularly that of $\mathrm{W}^{*}$-algebras.

The authors are grateful for the support that their research has received from Schweizerischer Nationalfonds/Fonds national suisse and from the United Kingdom Science and Engineering Research Council. Each author is also grateful for the hospitality received from the other's institution.

\section{Preliminaries}

Let $V$ be a complex vector space and let $C$ be a convex subset of $V$. A convex subset $E$ of $C$ is said to be a face of $C$ provided that, if $x$ is an element of $E$ such that $x=t x_{1}+(1-t) x_{2}$, where $x_{1}$ and $x_{2}$ lie in $C$ and $0<t<1$, then $x_{1}$ and $x_{2}$ lie in $E$. An element $x$ of $C$ for which $\{x\}$ is a face of $C$ is said to be an extreme point of $C$. Let $\tau$ be a total convex Hausdorff topology on $V$ and let $C$ be $\tau$-closed. Let $\mathscr{F}_{r}(C)$ denote the set of $\tau$-closed faces of $C$. Both $C$ and $\varnothing$ are elements of $\mathscr{F}_{\tau}(C)$ and the intersection of an arbitrary family of elements in $\mathscr{F}_{\tau}(C)$ again lies in $\mathscr{F}_{\tau}(C)$. Hence, with respect to the ordering by set inclusion $\mathscr{F}_{\tau}(C)$ forms a complete lattice. A subset $E$ of $C$ is said to be a $\tau$-exposed face of $C$ provided that there exists a $\tau$-continuous linear functional $f$ on $V$ and a real number $t$ such that, for all elements $x$ in $C \backslash E, \operatorname{Re} f(x)<t$ and, for all elements $x$ in $E, \operatorname{Re} f(x)=t$. Let $\mathscr{E}_{\tau}(C)$ denote the set of $\tau$-exposed faces of $C$. Clearly $\mathscr{E}_{\tau}(C)$ is contained in $\mathscr{F}_{\tau}(C)$ and the intersection of a finite number of elements of $\mathscr{E}_{\tau}(C)$ again lies in $\mathscr{E}_{\tau}(C)$. The intersection of an arbitrary family of elements of $\mathscr{E}_{\tau}(C)$ is said to be a $\tau$-semi-exposed face of $C$. Let $\mathscr{S}_{\tau}(C)$ denote the set of $\tau$-semiexposed faces of $C$. Clearly $\mathscr{E}_{\tau}(C)$ is contained in $\mathscr{S}_{\tau}(C)$ and the intersection of an 
arbitrary family of elements of $\mathscr{S}_{\tau}(C)$ again lies in $\mathscr{S}_{\tau}(C)$. Hence, with respect to the ordering by set inclusion $\mathscr{S}_{\tau}(C)$ forms a complete lattice and the infimum of a family of elements of $\mathscr{S}_{\tau}(C)$ coincides with its infimum when taken in $\mathscr{F}_{\tau}(C)$.

When $V$ is a complex Banach space with dual space $V^{*}$ the abbreviations $n$ and $w^{*}$ will be used for the norm topology of $V$ and the weak* topology of $V^{*}$. For each subset $E$ of the unit ball $V_{1}$ in $V$ let $E^{\prime}$ be the subset of the unit ball $V_{1}^{*}$ in $V^{*}$ defined by (1.1), and for each subset $F$ of $V_{1}^{*}$ let $F$, be the subset of $V_{1}$ defined by (1.2). The properties of these mappings are summarised in the following lemma, the proof of which is straightforward.

LeMma 2.1. (i) For subsets $D$ and $E$ of $V_{1}$ and $F$ and $G$ of $V_{1}^{*}$ and for any real number $t$,

$$
\begin{array}{cl}
\left(e^{i t} E\right)^{\prime}=e^{-i t} E^{\prime}, & \left(e^{i t} F\right),=e^{-i t} F, \\
E \subseteq\left(E^{\prime}\right), & F \subseteq(F)^{\prime} ;
\end{array}
$$

if $D \subseteq E$, then $E^{\prime} \subseteq D^{\prime}$ and if $F \subseteq G$, then $G, \subseteq F$,

(ii) A subset $E$ of $V_{1}$ lies in $\mathscr{S}_{n}\left(V_{1}\right)$ if and only if $E=\left(E^{\prime}\right)$, and a subset $F$ of $V_{1}^{*}$ lies in $\mathscr{S}_{w^{*}}\left(V_{1}^{*}\right)$ if and only if $F=(F)^{\prime}$.

(iii) The mappings $E \rightarrow E^{\prime}$ and $F \rightarrow F$, are anti-order isomorphisms between $\mathscr{S}_{n}\left(V_{1}\right)$ and $\mathscr{S}_{w^{*}}\left(V_{1}^{*}\right)$ and are inverses of each other.

For further details the reader is referred to $[3,10,11]$.

Recall that a real Jordan algebra $A$ which is also a Banach space such that, for all elements $a$ and $b$ in $A,\left\|a^{2}-b^{2}\right\| \leqslant \max \left\{\left\|a^{2}\right\|,\left\|b^{2}\right\|\right\}$ and $\left\|a^{2}\right\|=\|a\|^{2}$, is said to be a JBalgebra. The set $A^{+}$consisting of squares of elements of $A$ forms a norm closed cone in $A$. If $A$ is the dual of a Banach space $A_{*}$ then $A$ is said to be a JBW-algebra. In this case the cone $A^{+}$is weak* closed and $A$ is monotone complete. It follows that $A$ possesses a multiplicative unit $e$. With respect to the ordering induced from $A$ and the orthocomplementation $p \mapsto e-p$ the set of idempotents in $A$ forms a complete orthomodular lattice, two idempotents $p$ and $q$ being orthogonal if and only if $p \circ q=0$. Let $A_{*}^{+}$denote the norm closed cone in $A_{*}$ dual to $A^{+}$and let $K$ denote the set of elements of $A_{*}^{+}$of norm one. Elements of $A_{*}$ are precisely the normal linear functionals on $A$ and hence $K$ is said to be the set of normal states of $A$. A unital Jordan *-algebra $\mathfrak{U}$ which is also a complex Banach space such that, for all elements $a$ and $b$ in $\mathfrak{U},\left\|a^{*}\right\|=\|a\|,\|a \circ b\| \leqslant\|a\|\|b\|$ and $\|\{a a a\}\|=\|a\|^{3}$, where

$$
\{a b c\}=a \circ\left(b^{*} \circ c\right)-b^{*} \circ(c \circ a)+c \circ\left(a \circ b^{*}\right)
$$

is the Jordan triple product on $\mathfrak{A}$, is said to be a Jordan $\mathrm{C}^{*}$-algebra [33] or $\mathrm{JB}^{*}$-algebra [34]. Examples of $\mathrm{JB}^{*}$-algebras include all finite-dimensional formally real complex Jordan *-algebras and all unital $C^{*}$-algebras $A$ with Jordan product defined, for elements $a$ and $b$ in $\mathfrak{A}$, by $a \circ b=\frac{1}{2}(a b+b a)$. A Jordan $C^{*}$-algebra which is the dual of a Banach space $\mathfrak{U}_{*}$ is said to be a Jordan $\mathrm{W}^{*}$-algebra [8], or a $\mathrm{JBW}^{*}$-algebra [34]. The self-adjoint part $\mathfrak{U}_{\text {sa }}$ of a Jordan $C^{*}$-algebra (respectively Jordan $W^{*}$-algebra) is a JB-algebra (respectively JBW-algebra). Each JB-algebra (respectively JBW-algebra) is obtained in this manner. The correspondences so established are one to one. In addition, for a Jordan $W^{*}$-algebra $\mathfrak{U},\left(\mathfrak{U}_{\mathrm{sa}}\right)_{*}=\left(\mathfrak{U}_{*}\right)_{\mathrm{sa}}$. Notice that examples of Jordan $\mathrm{W}^{*}$-algebras are provided by $\mathrm{W}^{*}$-algebras equipped with the usual Jordan product. 
LEMMA 2.2. Let $\mathfrak{A}$ be a Jordan $\mathrm{C}^{*}$-algebra. Then the unit $e$ in $\mathfrak{U}$ is an extreme point of the unit ball $\mathfrak{U}_{1}$ in $\mathfrak{U}$.

Proof. Let $a$ and $b$ be elements of $\mathfrak{U}_{1}$ such that $e=\frac{1}{2} a+\frac{1}{2} b$. Putting $c=\frac{1}{2}\left(a+a^{*}\right)$ and $d=\frac{1}{2}\left(b+b^{*}\right)$, it follows that $e=\frac{1}{2} c+\frac{1}{2} d$. Let $B$ be the sub-JB-algebra of the selfadjoint part $\mathfrak{U}_{\mathrm{sa}}$ of $\mathfrak{U}$ generated by $c, d$, and $e$. Then, by [33, Proposition 2.1], $B$ may be regarded as a JB-algebra of bounded self-adjoint operators on a complex Hilbert space and [29, Proposition 1.6.1] shows that $c=d=e$. Hence $a^{*}=2 e-a$, and applying [33, Corollary 2.2] it follows that $a=e$, as required.

LEMMA 2.3. Let $\mathfrak{U}$ be a Jordan $\mathrm{W}^{*}$-algebra with unit $e$ and let $x$ be an element of norm one in the predual $\mathfrak{U}_{*}$ of $\mathfrak{U}$ such that $x(e)=1$. Then $x$ is a normal state of $\mathfrak{U}_{\mathrm{sa}}$.

Proof. Let $a$ be an element of $\mathfrak{A}_{\mathrm{sa}}$ and suppose that $x(a)=s+i t$, where $s$ and $t$ are real. Let $W(a, e)$ be the smallest sub-JBW-algebra of $\mathfrak{U}_{\mathrm{sa}}$ containing $a$ and $e$, and let $\mathfrak{W}(a, e)=W(a, e)+i W(a, e)$. Then, by [7, Lemma 3.2], $\mathfrak{B}(a, e)$ is a commutative $\mathrm{W}^{*}$-algebra. Applying [29, Proposition 1.5.2] to the restriction of $x$ to $\mathfrak{M}(a, e)$, it follows that $t=0$. The proof of the corresponding result for $\mathrm{W}^{*}$-algebras can also be applied in this case to show that $x$ is positive.

For further details of the theory of JBW-algebras and Jordan $\mathrm{W}^{*}$-algebras the reader is referred to $[1,2,6,7,19,23,30]$.

A complex vector space $\mathfrak{U}$ equipped with a triple product $(a, b, c) \rightarrow\{a b c\}$ from $\mathfrak{U} \times \mathfrak{U} \times \mathfrak{U}$ to $\mathfrak{U}$ which is symmetric and linear in the first and third variables, conjugate linear in the second variable and satisfies the identity

$$
\begin{aligned}
{[D(a, b), D(c, d)] } & =D(\{a b c\}, d)-D(c,\{d a b\}) \\
& =D(a,\{b c d\})-D(\{c d a\}, b),
\end{aligned}
$$

where [,] denotes the commutator and $D$ is the mapping from $\mathfrak{U} \times \mathfrak{U}$ to the space of linear operators on $\mathfrak{U}$ defined by $D(a, b) c=\{a b c\}$, is said to be a Jordan triple. When $\mathfrak{U}$ is also a Banach space such that $D$ is continuous from $\mathfrak{U} \times \mathfrak{U}$ to the Banach space $\mathfrak{L}(\mathfrak{U})$ of bounded linear operators on $\mathfrak{U}$ and, for each element $a$ in $\mathfrak{A}, D(a, a)$ is hermitean with non-negative spectrum and satisfies $\|D(a, a)\|=\|a\|^{2}$, then $\mathscr{A}$ is said to be a JB*-triple. In this event it can be shown that, for all elements $a, b$ and $c$ in $\mathfrak{U}$, $\|\{a b c\}\| \leqslant\|a\|\|b\|\|c\|$ and $\|\{a a a\}\|=\|a\|^{3}$ [17]. Let $H_{1}$ and $H_{2}$ be complex Hilbert spaces. A norm-closed subspace $\mathfrak{U}$ of the Banach space $\mathfrak{L}\left(H_{1}, H_{2}\right)$ of bounded linear operators from $H_{1}$ to $H_{2}$ with the property that for each element $a$ in $\mathfrak{U}$, the bounded operator $a a^{*} a$ also lies in $\mathcal{U}$, is said to be a $\mathrm{J}^{*}$-algebra [21]. With respect to the triple product $\{a b c\}=\frac{1}{2}\left(a b^{*} c+c b^{*} a\right)$ a $\mathrm{J}^{*}$-algebra is a $\mathrm{JB}^{*}$-triple. Further examples of $\mathrm{JB}^{*}$ triples are provided by Jordan $C^{*}$-algebras with the triple product (2.1). In particular, every $\mathrm{C}^{*}$-algebra is a $\mathrm{JB}^{*}$-triple. A $\mathrm{JB}^{*}$-triple $\mathfrak{A}$ which is the dual of a Banach space $\mathfrak{U}_{*}$ is said to be a $\mathrm{JBW}^{*}$-triple. In this case the predual is unique and the triple product is separately weak* continuous $[4,5]$. Weak* closed $\mathrm{J}^{*}$-algebras and Jordan $\mathrm{W}^{*}$ algebras provide examples of $\mathrm{JBW}^{*}$-triples. In particular, $\mathrm{W}^{*}$-algebras are $\mathrm{JBW}^{*}$ triples. An element $u$ in a $\mathrm{JB}^{*}$-triple $\mathfrak{A}$ is said to be a tripotent if $\{u \boldsymbol{u} u=u$. The set of tripotents in $\mathfrak{U}$ is denoted by $\mathscr{U}(\mathfrak{U})$. Notice that if $\mathfrak{U}$ is a $J^{*}$-algebra then $\mathscr{U}(\mathfrak{U})$ is 
the set of partial isometries in $\mathfrak{U}$. For each tripotent $u$, the operators $Q(u), P_{j}(u)$, $j=0,1,2$, are defined by

$$
\begin{gathered}
Q(u) a=\{u a u\}, \quad P_{2}(u)=Q(u)^{2}, \quad P_{1}(u)=2\left(D(u, u)-Q(u)^{2}\right), \\
P_{0}(u)=I-2 D(u, u)+Q(u)^{2} .
\end{gathered}
$$

The operators $P_{j}(u), j=0,1,2$, are projections onto the eigenspaces $\mathfrak{U}_{j}(u)$ of $D(u, u)$ corresponding to the eigenvalues $\frac{1}{2} j$ and

$$
\mathfrak{U}=\mathfrak{U}_{0}(u) \oplus \mathfrak{U}_{1}(u) \oplus \mathfrak{U}_{2}(u)
$$

is the Peirce decomposition of $\mathfrak{A}$ relative to $u$. For $j, k, l=0,1,2, \mathfrak{A}_{j}(u)$ is a sub-JB*triple of $\mathfrak{U}$ such that

$$
\left\{\mathfrak{U}_{j}(u) \mathfrak{U}_{k}(u) \mathfrak{U}_{l}(u)\right\} \subseteq \mathfrak{U}_{j-k+l}(u)
$$

when $j-k+l=0,1$ or 2 and is $\{0\}$ otherwise, and

$$
\left\{\mathfrak{U}_{2}(u) \mathfrak{A}_{0}(u) \mathfrak{A}\right\}=\left\{\mathfrak{U}_{0}(u) \mathfrak{U}_{2}(u) \mathfrak{A}\right\}=\{0\} .
$$

Moreover, $\mathfrak{U}_{2}(u)$ is a Jordan $C^{*}$-algebra with product $(a, b) \rightarrow\{a u b\}$, unit $u$, and involution $a \rightarrow\{u a u\}$. If $\mathscr{U}$ is a $\mathrm{J}^{*}$-algebra and $u$ is a partial isometry in $\mathscr{U}$, with initial and final projections $u^{*} u$ and $u u^{*}$, respectively, then

$$
\begin{gathered}
P_{2}(u) a=u u^{*} a u^{*} u, \quad P_{0}(u) a=\left(1-u u^{*}\right) a\left(1-u^{*} u\right), \\
P_{1}(u) a=u u^{*} a\left(1-u^{*} u\right)+\left(1-u u^{*}\right) a u^{*} u .
\end{gathered}
$$

If $\mathfrak{U}$ is a $\mathrm{JBW}^{*}$-triple then the operators $D(a, b), Q(u), P_{j}(u), j=0,1,2$, are all weak* continuous and $\mathfrak{U}_{j}(u), j=0,1,2$, are sub-JBW*-triples of $\mathfrak{U}$ with $\mathfrak{U}_{2}(u)$ a Jordan $\mathrm{W}^{*}$ algebra. A pair $u, v$ of elements of $\mathscr{U}(\mathfrak{U})$ is said to be orthogonal if one of the following equivalent conditions holds:

$$
\begin{array}{cl}
D(u, v)=0 ; & D(v, u)=0 \\
\{u u v\}=0 ; & \{v v u\}=0 \\
v \in \mathfrak{U}_{0}(u) ; & u \in \mathfrak{U}_{0}(v) .
\end{array}
$$

For two elements $u$ and $v$ of $\mathscr{U}(\mathfrak{U})$, write $u \leqslant v$ if $v-u$ is a tripotent orthogonal to $u$ or, equivalently, if

$$
P_{2}(u) v=u \text {. }
$$

This defines a partial ordering on $\mathscr{U}(\mathfrak{A})$. Notice that, if $u \leqslant v$ then, since $u$ and $v-u$ are orthogonal,

$$
\{v u v\}=-\{u u u\}+2\{v u u\}=-u+2\{v-u u u\}+2 u=u
$$

and it follows that

$$
P_{2}(v) u=u
$$

LEMMA 2.4. Let $\mathfrak{U}$ be a $\mathrm{JBW}^{*}$-triple and let $v$ be an element of $\mathscr{U}(\mathfrak{U})$.

(i) An element $u$ in $\mathfrak{U}$ is a tripotent such that $u \leqslant v$ if and only if $u$ is an idempotent in the JBW-algebra $\mathfrak{A}_{2}(v)_{\mathrm{sa}}$.

(ii) Let $u_{1}$ and $u_{2}$ be elements of $\mathscr{U}(\mathfrak{U})$ such that $u_{1}, u_{2} \leqslant v$. Then $u_{1} \leqslant u_{2}$ if and only if $u_{2}-u_{1}$ lies in $\mathfrak{U}_{2}(v)_{\mathrm{sa}}^{+}$. 
Proof. (i) Notice that, by (2.4), in $\mathfrak{U}_{2}(v)$,

$$
u \circ u=\{u v u\}=\{\{u u u\} v\{u u u\}\}=\left\{u P_{2}(u) v u\right\}=u .
$$

Moreover, since $v-u$ and $u$ are orthogonal,

$$
u^{*}=\{v u v\}=-\{u \quad u u\}+2\{v u u\}=-u+2\{v-u u u\}+2 u=u .
$$

Conversely, if $u$ is an element of $\mathfrak{A}_{2}(v)$ such that $\{v u v\}=u$ and $\{u v u\}=u$, then $\{u u u\}=2 u \circ(u \circ u)-u^{2} \circ u=u$.

(ii) Suppose that $u_{1} \leqslant u_{2} \leqslant v$. Then, applying (2.2) and (2.3),

$$
\begin{aligned}
\left\{u_{1} v u_{2}\right\} & =\left\{\left\{u_{1} u_{1} u_{1}\right\} v u_{2}\right\}=D\left(u_{2}, v\right) D\left(u_{1}, u_{1}\right) u_{1} \\
& =D\left(u_{2},\left\{v u_{1} u_{1}\right\}\right) u_{1}-D\left(\left\{u_{1} u_{1} u_{2}\right\}, v\right) u_{1}+D\left(u_{1}, u_{1}\right) D\left(u_{2}, v\right) u_{1} \\
& =D\left(u_{1}, u_{1}\right)\left\{u_{1} v u_{2}\right\} .
\end{aligned}
$$

It follows that $\left\{u_{1} v u_{2}\right\}$ is an element of $\mathfrak{A}_{2}\left(u_{1}\right)$ and hence that

$$
\begin{aligned}
u_{1} \circ u_{2} & =\left\{u_{1} v u_{2}\right\}=\left\{u_{1}\left\{u_{1} v u_{2}\right\} u_{1}\right\}=D\left(u_{1},\left\{u_{1} v u_{2}\right\}\right) u_{1} \\
& =D\left(\left\{v u_{2} u_{1}\right\}, u_{1}\right) u_{1}+D\left(u_{1}, u_{1}\right) D\left(v, u_{2}\right) u_{1}-D\left(v, u_{2}\right) D\left(u_{1}, u_{1}\right) u_{1} \\
& =u_{1}+u_{1}-u_{1}=u_{1} .
\end{aligned}
$$

Hence $u_{1} \leqslant u_{2}$ in $\mathfrak{U}_{2}(v)_{\mathrm{sa}}$. Conversely, suppose that this is the case. Then, since $u_{1}$ is orthogonal to $v-u_{1}$,

$$
\left\{\begin{array}{lll}
u_{1} & u_{1} & u_{2}
\end{array}\right\}=\left\{\begin{array}{lll}
u_{1} & v & u_{2}
\end{array}\right\}=u_{1},
$$

which implies that $D\left(u_{1}, u_{1}\right)\left(u_{1}-u_{2}\right)=0$ and $u_{1}-u_{2}$ lies in $\mathfrak{U}_{0}\left(u_{1}\right)$ as required.

For further details of the properties of $\mathrm{JB}^{*}$-triples and $\mathrm{JBW}^{*}$-triples the reader is referred to $[4,5,12$ to $18,22,26,31,32]$.

\section{Supports of elements of a $\mathrm{JBW}^{*}$-triple}

Throughout this section $\mathfrak{U}$ denotes a $\mathrm{JBW}^{*}$-triple with predual $\mathfrak{U}_{*}$ and $\mathscr{U}(\mathfrak{U})$ denotes the set of tripotents in $\mathfrak{U}$.

Let $a$ be an element of $\mathfrak{U}$ of norm one and let $\mathfrak{M}(a)$ be the smallest sub-JBW*triple of $\mathcal{U}$ containing $a$. Notice that $\mathfrak{W}(a)$ is the weak* closure of the linear span of the elements $a^{2 n+1}$ of $\mathfrak{A}$ defined inductively by

$$
a^{3}=\{a \quad a \quad a\}, \quad a^{2 n+1}=\left\{a a^{2 n-1} a\right\} .
$$

Then, by $[17$, Corollary $6 ; 21$, Theorem $3.2 ; 13], \mathfrak{W}(a)$ is isomorphic as a $\mathrm{JBW}^{*}$-triple to a commutative $\mathrm{W}^{*}$-algebra $L_{\infty}(\Omega, \mu)$. By slight abuse of notation, $\mathfrak{B}(a)$ and $L_{\infty}(\Omega, \mu)$ will be identified. Consequently, if

$$
a=r(a)|a|, \quad|a|=r(a)^{*} a
$$

is the polar decomposition of $a$ in $L_{\infty}(\Omega, \mu)$, then

$$
a^{2 n+1}=a|a|^{2 n}=r(a)|a|^{2 n+1}
$$

and $r(a)$ is a partial isometry in $L_{\infty}(\Omega, \mu)$, hence an element of $\mathscr{U}(\mathfrak{U})$ such that

$$
\{r(a) r(a) a\}=\{r(a) a r(a)\}=a .
$$

The tripotent $r(a)$ is said to be the support of $a$. Clearly $a$ can be regarded as an element of the JBW-algebra $\mathfrak{A}_{2}(r(a))_{\mathrm{sa}}$. 
LEMMA 3.1. The complexification of the smallest sub-JBW-algebra $W(a)$ of $\mathfrak{U}_{2}(r(a))_{\mathrm{sa}}$ containing a coincides with $\mathfrak{W}(a)$, and $r(a)$ is the unit in $W(a)$.

Proof. First observe that every Jordan *-subalgebra of $\mathfrak{U}_{2}(r(a))$ is a subtriple, and hence that $\mathfrak{W}(a)$ is contained in $W(a)+i W(a)$. Moreover, since $\mathfrak{W}(a)$ is a subtriple containing $r(a)$, for all integers $n$ and $m,\left\{a^{2 n+1} r(a) a^{2 m+1}\right\}$ and $\left\{r(a) a^{2 n+1} r(a)\right\}$ are elements of $\mathfrak{B}(a)$. Using the weak* continuity properties of the triple product it follows that $\mathfrak{M}(a)$ is a Jordan $\mathrm{W}^{*}$-subalgebra of $\mathfrak{U}_{2}(r(a))$ and therefore coincides with $W(a)+i W(a)$.

By spectral theory there exists a sequence $\left(q_{n}\right)$ of real odd polynomials of degree $n$ on $[0,1]$ such that the sequence $\left(q_{n}(|a|)\right)$ converges in the weak* topology of $L_{\infty}(\Omega, \mu)$ to $r(a)^{*} r(a)$, the range projection of $|a|$. Notice that, by $(3.2), q_{n}(a)=r(a) q_{n}(|a|)$ and, by the weak* continuity of multiplication, the sequence $\left(q_{n}(a)\right)$ converges in the weak* topology to $r(a)$. Let $e^{|a|}$ be the spectral measure on $[0,1]$ corresponding to $|a|$ with range in $L_{\infty}(\Omega, \mu)$. Then, as in [21, Theorem 3.2], $u^{a}$, defined for each Borel subset $M$ of $[0,1]$ by $u^{a}(M)=r(a) e^{|a|}(M)$, is a $\mathscr{U}(\mathfrak{U})$-valued measure on $[0,1]$ such that

$$
a=\int_{0}^{1} t d u_{t}^{a}
$$

A simple calculation shows that, for all Borel subsets $M$ of $[0,1], u^{a}(M) \leqslant r(a)$ and, by using Lemma 2.4 , that (3.3) is precisely the spectral resolution of $a$ in the JBWalgebra $\mathfrak{U}_{2}(r(a))_{\mathrm{sa}}$. It follows that, in $\mathfrak{U}_{2}(r(a))_{\mathrm{sa}}, 0 \leqslant a \leqslant r(a)$. The unit in $W(a)$ is the range idempotent of $a$ and can be regarded as the weak* limit of the sequence $\left(q_{n}(a)\right)$ in $\mathfrak{U}_{2}(r(a))_{\mathrm{sa}}$. Since, in $\mathfrak{U}_{2}(r(a))$, odd powers of $a$ defined by the Jordan product of $\mathfrak{U}_{2}(r(a))$ and by the triple product of $\mathfrak{U}$ coincide it follows that $r(a)$ is precisely the unit in $W(a)$.

Notice that the fact that $\mathscr{U}(\mathfrak{U})$ contains a non-zero element is a consequence of this result. The next result describes a further property of the partially ordered set $\mathscr{U}(\mathfrak{U})$.

LEMMA 3.2. The mapping $u \rightarrow\{u\}$, is an order isomorphism from $\mathscr{U}(\mathfrak{U})$ onto the partially ordered set $\mathscr{E}_{n}\left(\mathfrak{U}_{* 1}\right) \backslash \mathfrak{U}_{* 1}$ of norm-exposed faces of $\mathfrak{U}_{*_{1}}$ with $\mathfrak{U}_{*_{1}}$ excluded.

Proof. Let $u$ and $v$ be elements of $\mathscr{U}(\mathfrak{U})$ with $u \leqslant v$. Notice that, for each element $x$ in $\{u\}$,

$$
\left|\left(P_{2}(u)^{*} x\right)(u)\right|=\left|x\left(P_{2}(u) u\right)\right|=|x(u)|=1
$$

and therefore $\left\|P_{2}(u)^{*} x\right\|=\|x\|$. It follows from [16, Proposition 1] that $P_{2}(u)^{*} x=x$. Then, by (2.3),

$$
x(v)=P_{2}(u)^{*} x(v)=x\left(P_{2}(u) v\right)=x(u)=1 .
$$

Hence $\{u\}, \subseteq\{v\}$, Conversely, suppose that $u$ and $v$ are elements of $\mathscr{U}(\mathfrak{X})$ such that $\{u\}, \subseteq\{v\}$. Elements of $\{u\}$, may be regarded as linear functionals of norm one in the predual $\mathfrak{U}_{2}(u)_{*}$ of the JBW*-algebra $\mathfrak{A}_{2}(u)$. By Lemma 2.3, $\{u\}$, can be identified with the normal state space of $\mathfrak{U}_{2}(u)$. However, $P_{2}(u) v$ is an element of $\mathfrak{U}_{2}(u)$ such that, for each element $x$ of its normal state space,

$$
x\left(P_{2}(u) v\right)=P_{2}(u)^{*} x(v)=x(v)=1 .
$$


It follows that $P_{2}(u) v$ is the unit $u$ in $\mathfrak{A}_{2}(u)$ and hence that $u \leqslant v$. It remains to show that the mapping $u \rightarrow\{u\}$, is surjective. Let $a$ be an element of $\mathfrak{A}$ of norm one. Then, using Lemma 3.1, as in [16, Proposition 8], there exists an element $u(a)$ in $\mathscr{U}(\mathfrak{U})$ such that $\{a\}$, and $\{u(a)\}$, coincide. Finally, observe that $\{0\}$, is the empty set and the proof is complete.

COROLlaRY 3.3. For each element $u$ in $\mathscr{U}(\mathfrak{H})$ the smallest weak ${ }^{*}$-semi-exposed face $\{u\}$, of $\mathfrak{A}_{1}$ containing $u$ is $u+\mathfrak{A}_{0}(u)_{1}$.

Proof. By [16, Lemma 1.3(a)], for each element $a$ in $\mathfrak{A}_{0}(u)_{1}, u+a$ lies in $\mathfrak{A}_{1}$. Moreover, for each element $x$ in $\{u\}$,

$$
x(u+a)=P_{2}(u)^{*} x\left(u+P_{0}(u) a\right)=x(u)=1
$$

and $u+\mathfrak{Q}_{0}(u)_{1}$ is contained in $\{u\},{ }^{\prime}$. Conversely, if $a$ is an element of $\{u\}$, then $P_{2}(u) a$ is an element of $\mathfrak{O}_{2}(u)$ which takes the value one on its normal state space, and hence $P_{2}(u) a=u$. By [16, Lemma 1.6], $P_{1}(u) a=0$, and it follows that $a=u+P_{0}(u) a$. Since $P_{0}(u)$ is a contraction, $a$ lies in $u+\mathfrak{U}_{0}(u)_{1}$ and the proof is complete.

For each element $a$ of $\mathfrak{U}$ of norm one let $u(a)$ be the unique element of $\mathscr{U}(\mathfrak{U})$, defined in Lemma 3.2, with the property that $\{a\}$, and $\{u(a)\}$, coincide. The next result gives alternative characterisations of the tripotent $u(a)$ associated with $a$.

Lemma 3.4. (i) The tripotent $u(a)$ is the greatest element of the subset $\mathscr{S}$ of $\mathscr{U}(\mathfrak{H})$ consisting of elements $u$ such that $\{u a u\}=u$.

(ii) The tripotent $u(a)$ is the limit in the weak topology of the sequence $\left(a^{2 n+1}\right)$.

(iii) If $u$ is an element of $\mathscr{S}$ such that $\left\|P_{0}(u) a\right\|<1$, then $u=u(a)$.

Proof. Notice that, as in the proof of Lemma 3.2, $P_{2}(u(a)) a=u(a)$ and hence $u(a)$ is an element of $\mathscr{S}$. If $u$ is an arbitrary element of $\mathscr{S}$ then $P_{2}(u) a=u$ and $\{u\}, \subseteq\{a\},=\{u(a)\}$, By Lemma 3.2, $u \leqslant u(a)$ and the proof of (i) is complete. To prove (ii) the notation used in the proof of Lemma 3.2 is maintained. By spectral theory in $L_{\infty}(\Omega, \mu)$, the sequence $\left(b_{n}\right)$ defined by

$$
b_{n}=r(a)^{*} r(a)-\left(r(a)^{*} r(a)-|a|\right)^{2 n+1}
$$

converges in the weak* topology to $r(a)^{*} r(a)$. Hence, the sequence $\left(|a|^{2 n+1}\right)$ converges in the weak* topology to $r(a)^{*} r(a)-r$, where $r$ is the range idempotent of the positive element $r(a)^{*} r(a)-|a|$. By (3.2), the sequence $\left(a^{2 n+1}\right)$ converges in the weak* topology to the element $w$, where $w=r(a) r(a)^{*} r(a)-r(a) r=r(a)-r(a) r$. Notice that

$$
\{r(a) r r(a) r r(a) r\}=r(a) r, \quad\{r(a) r r(a) r(a) r\}=r(a) r,
$$

and $r(a) r$ is an idempotent in $\mathfrak{U}_{2}(r(a))_{\mathrm{sa}}$. Therefore, $w$ is an idempotent in $\mathfrak{A}_{2}(r(a))_{\mathrm{sa}}$ and, by Lemma 2.4, $w$ is an element of $\mathscr{U}(\mathfrak{A})$ such that $w \leqslant r(a)$. Moreover,

$$
\begin{aligned}
\{w a w\} & =(r(a)-r(a) r) a^{*}(r(a)-r(a) r)=(r(a)-r(a) r)(|a|-|a| r) \\
& =(r(a)-r(a) r)\left(|a|-r(a)^{*} r(a) r+r(a)^{*} r(a)-|a|\right) \\
& =w .
\end{aligned}
$$

It follows from (i) that $w \leqslant u(a)$. Regarded as elements of $\mathfrak{A}_{2}(r(a))_{\mathrm{sa}}^{+}$, the sequence $\left(a^{2 n+1}\right)$ decreases with infimum $w$ and therefore converges to $w$ in the weak* topology 
of $\mathfrak{U}_{2}(r(a))$. The weak* continuity of the Peirce projections shows that the convergence is also in the weak* topology of $\mathfrak{U}$. As in the proof of Corollary 3.3,

$$
a=u(a)+P_{0}(u(a)) a,
$$

from which it follows that

$$
a^{2 n+1}=u(a)+\left(P_{0}(u(a)) a\right)^{2 n+1} .
$$

If $x$ is an element of $\{u(a)\}$, since $P_{2}(u(a))^{*} x=x$ and $\mathfrak{U}_{0}(u(a))$ is a subtriple,

$$
x\left(a^{2 n+1}\right)=1+x\left(\left(P_{0}(u(a)) a\right)^{2 n+1}=1 .\right.
$$

Therefore $x(w)=1$ and $x$ lies in $\{w\}$, By Lemma 3.2, $u(a) \leqslant w$, and therefore $u(a)$ and $w$ coincide. To prove (iii) suppose that $u$ is an element of $\mathscr{S}$. Then $a=u+P_{0}(u) a$ and $a^{2 n+1}=u+\left(P_{0}(u) a\right)^{2 n+1}$. If $\left\|P_{0}(u) a\right\|<1$ then the sequence $\left(a^{2 n+1}\right)$ converges in norm and hence in the weak* topology to $u$. From above $u=u(a)$.

The next result is the principal technical lemma needed in $\$ 4$ to describe the set of weak* closed faces of $\mathfrak{U}_{1}$.

LEMMA 3.5. (i) The tripotent $u(a)$ is contained in the weak* closure $\overline{\text { face }}^{w^{*}}$ of the smallest face face $a$ of $\mathfrak{U}_{1}$ containing $a$.

(ii) The weak* semi-exposed face $u(a)+\mathfrak{A}_{0}(u(a))_{1}$ of $\mathfrak{U}_{1}$ coincides with the smallest face face $\left(\overline{\text { face } a}{ }^{w^{*}}\right)$ of $\mathfrak{U}_{1}$ containing $\overline{\text { face } a} w^{*}$.

Proof. Maintaining the notation used above, define the sequence $\left(b_{n}\right)$ of elements of $L_{\infty}(\Omega, \mu)$ by

$$
b_{n}=r(a)^{*} r(a)-\left(r(a)^{*} r(a)-\left(r(a)^{*} r(a)-|a|\right)\right)^{2 n+1} .
$$

Notice that the sequence $\left(b_{n}\right)$ lies in $\mathfrak{U}_{1}$ and, by spectral theory,

$$
\begin{gathered}
0 \leqslant b_{n} \leqslant(2 n+1)\left(r(a)^{*} r(a)-|a|\right), \\
\left\|(2 n+1)\left(r(a)^{*} r(a)-|a|\right)-b_{n}\right\|<2 n .
\end{gathered}
$$

Moreover,

$$
r(a)^{*} r(a)-|a|=(2 n+1)^{-1} b_{n}+2 n(2 n+1)^{-1} c_{n},
$$

where

$$
c_{n}=(2 n)^{-1}\left((2 n+1)\left(r(a)^{*} r(a)-|a|\right)-b_{n}\right)
$$

Hence,

$$
|a|=(2 n+1)^{-1}\left(r(a)^{*} r(a)-b_{n}\right)+2 n(2 n+1)^{-1}\left(r(a)^{*} r(a)-c_{n}\right)
$$

a convex combination of elements in $L_{\infty}(\Omega, \mu)_{1}$. Therefore,

$$
a=r(a)|a|=(2 n+1)^{-1}\left(r(a)-r(a) b_{n}\right)+2 n(2 n+1)^{-1}\left(r(a)-r(a) c_{n}\right) \text {, }
$$

a convex combination of elements of $\mathfrak{U}_{1}$. Moreover,

$$
r(a)-r(a) b_{n}=r(a)|a|^{2 n+1}=a^{2 n+1} \text {. }
$$

It follows that $a^{2 n+1}$ is an element of face $a$ and, by Lemma 3.4(iii), that $u(a)$ lies in $\overline{\text { face } a}{ }^{w^{*}}$.

Now let $b$ be an element of $\mathfrak{U}_{0}(u(a))_{1}$. By [16, Lemma 1.3], $u(a) \pm b$ are elements of $\mathfrak{U}_{1}$ and, since $u(a)=\frac{1}{2}(u(a)+b)+\frac{1}{2}(u(a)-b), u(a) \pm b$ lie in face $u(a)$. Therefore, $u(a)+\mathfrak{U}_{0}(u(a))_{1}$ is contained in face $\left(\overline{\text { face } a}{ }^{w^{*}}\right)$. However, by Corollary 3.3 and Lemma 
3.4, $a$ lies in the weak*-semi-exposed face $u(a)+\mathfrak{H}_{0}(u(a))_{1}$ of $\mathfrak{A}_{1}$. Therefore, face $a$, face $a w^{*}$, and face $\left(\overline{\text { face } a} w^{*}\right)$ are contained in $u(a)+\mathfrak{Q}_{0}(u(a))_{1}$ and the proof is complete.

Let $x$ be an element of $\mathfrak{A}_{* 1}$ of norm one. Then, by [16, Proposition 2], there exists a unique tripotent $e(x)$ in $\mathfrak{A}$ such that $P_{2}(e(x))^{*} x=x$ and the restriction of $x$ to the Jordan $\mathrm{W}^{*}$-algebra $\mathfrak{A}_{2}(e(x))$ is a faithful normal state.

Lemma 3.6. The tripotent $e(x)$ is the least element in $\{x\}^{\prime} \cap \mathscr{U}(\mathfrak{U})$.

Proof. Notice that since $x(e(x))=1, e(x)$ is a tripotent in $\{x\}^{\prime}$. Let $u$ be a tripotent in $\{x\}^{\prime}$, from which it follows that $x \in\{x\}^{\prime}, \subseteq\{u\}$, Therefore, $x$ is a normal state of the JBW-algebra $\mathfrak{A}_{2}(u)_{\mathrm{sa}}$. The support idempotent of $x$ in $\mathfrak{A}_{2}(u)_{\mathrm{sa}}$ has the same properties as $e(x)$ and therefore, by uniqueness, coincides with $e(x)$. It follows that $e(x) \leqslant u$ as required.

\section{Main results}

The main results of the paper are proved in this section. The notation of $\S 3$ is retained.

LEMMA 4.1. The mapping $u \rightarrow\{u\}$, is an order isomorphism from $\mathscr{U}(\mathfrak{A})$ onto the partially ordered set $\mathscr{S}_{n}\left(\mathfrak{A}_{*_{1}}\right) \backslash \mathfrak{A}_{*_{1}}$ of norm-semi-exposed faces of $\mathfrak{A}_{*_{1}}$ with $\mathfrak{A}_{*_{1}}$ excluded.

Proof. By Lemma 3.2 it is only required to show that every norm-semi-exposed face of $\mathfrak{A}_{*_{1}}$ is norm-exposed. First, let $F$ be a norm-semi-exposed face of $\mathfrak{U}_{*_{1}}$ different from $\mathfrak{U}_{* 1}$. There exists a family $\left\{u_{\delta}: \delta \in \Delta\right\}$ of elements of $\mathscr{U}(\mathfrak{U})$ such that $F=\bigcap\left\{\left\{u_{\delta}\right\},: \delta \in \Delta\right\}$. Let $\Delta^{f}$ be the set of non-empty finite subsets of $\Delta$ directed by set inclusion.For each element $j$ in $\Delta^{f}$, let $u_{j}$ be the unique tripotent such that $\left\{u_{j}\right\},=\bigcap\left\{\left\{u_{j}\right\},: \delta \in j\right\}$. It follows from Lemma 3.2 that if $j_{1} \supset j_{2}$ then $u_{j_{1}} \leqslant u_{j_{j}}$. Therefore $\left\{u_{j}: j \in \Delta^{f}\right\}$ forms a decreasing net in $\mathscr{U}(\mathfrak{H})$. Fix $j_{0}$ in $\Delta^{f}$ and let $\Delta^{f_{0}} \stackrel{u_{1}}{=}\left\{j: j \in \Delta^{f}, j_{0} \subseteq j\right\}$. Then $\left\{u_{j}: j \in \Delta^{f_{0}}\right\}$ forms a decreasing subnet in $\mathscr{U}(\mathfrak{Q})$. Therefore, by Lemma 2.4 , when regarded as idempotents in the JBW-algebra $\mathfrak{A}_{2}\left(u_{j_{0}}\right)_{\mathrm{s}}$, the decreasing net $\left\{u_{j}: j \in \Delta^{f_{0}}\right\}$ converges in the weak* topology to its infimum, an idempotent $u_{0}$. By Lemma 2.4, $u_{0}$ lies in $\mathscr{U}(\mathfrak{U})$. Moreover, the net $\left\{u_{j}: j \in \Delta^{f_{0}}\right\}$ converges to $u_{0}$ in the weak* topology of $\mathscr{U}$. Suppose that $j$ is any element of $\Delta^{f}$. Then the union $j_{1}$ of $j$ and $j_{0}$ contains $j$ and therefore $u_{0} \leqslant u_{j_{1}} \leqslant u_{j}$. Hence $u_{0}$ is a lower bound for $\left\{u_{j}: j \in \Delta^{\prime}\right\}$. If the tripotent $v_{0}$ is a further lower bound for $\left\{u_{j}: j \in \Delta^{f}\right\}$ then $v_{0}$ is also a lower bound for $\left\{u_{j}: j \in \Delta^{f_{0}}\right\}$ and $v_{0} \leqslant u_{0}$. It follows that $u_{0}$ is the infimum of the net $\left\{u_{j}: j \in \Delta^{\prime}\right\}$ and hence that, for all $\delta$ in $\Delta, u_{0} \leqslant u_{\delta}$. Therefore,

$$
\left.\left\{u_{0}\right\}, \subseteq \bigcap\left\{u_{\delta}\right\},: \delta \in \Delta\right\}=F .
$$

Now let $x$ be an element of $F$. Then, for all elements $\delta$ in $\Delta, x\left(u_{\delta}\right)=1$, and so for all elements $j$ in $\Delta^{f_{0}}, x\left(u_{j}\right)=1$. But, since $\left\{u_{j}: j \in \Delta^{f_{0}}\right\}$ converges in the weak* topology to $u_{0}$, it follows that $x\left(u_{0}\right)=1$ and $x$ lies in $\left\{u_{0}\right\}$. Therefore $F$ and $\left\{u_{0}\right\}$, coincide as required. 
COROLLARY 4.2. Let $x$ be an element of $\mathfrak{A}_{* 1}$ of norm one. Then $\{e(x)\}_{,}=\{x\}^{\prime}$,.

Proof. By Lemma 3.6, $\{x\}^{\prime}, \subseteq\{e(x)\}$. However, $\{x\}^{\prime}$, is a norm-semi-exposed face of $\mathfrak{A}_{* 1}$ different from $\mathfrak{A}_{* 1}$ and, by Lemma 4.1, there exists an element $u$ in $\mathscr{U}(\mathfrak{H})$ such that $\{x\}^{\prime},=\{u\}$. Hence, by Lemma 3.2, $u \leqslant e(x)$. But, $u \in\{u\}^{\prime},=\{x\}^{\prime},^{\prime}=\{x\}^{\prime}$ and, by Lemma 3.7, $e(x) \leqslant u$. This completes the proof.

Let $\mathscr{U}(\mathfrak{U})^{\sim}$ be the disjoint union of $\mathscr{U}(\mathfrak{U})$ with a one point set $\{\omega\}$, and define a relation on $\mathscr{U}(A)^{\sim}$ by writing $u \leqslant v$ if both $u$ and $v$ lie in $\mathscr{U}(\mathfrak{I})$ and $u \leqslant v$ in the ordering of $\mathscr{U}(\mathfrak{A})$ or if $u$ is an arbitrary element of $\mathscr{U}(\mathfrak{I})^{\sim}$ and $v$ and $\omega$ coincide. It is easily seen that this defines a partial ordering on $\mathscr{U}(\mathfrak{R})^{\sim}$ which extends that on $\mathscr{U}(\mathfrak{U})$. Define $\{\omega\}$, to be equal to $\mathfrak{H}_{* 1}$ in which case $\{\omega\}$, is the empty set.

COROLLARY 4.3. With respect to the ordering defined above $\mathscr{U}(\mathfrak{U})^{\sim}$ forms a complete lattice.

Proof. Clearly the partially ordered set $\mathscr{U}(\mathfrak{H})^{\sim}$ has the least element 0 and greatest element $\omega$. Moreover, by Lemma 4.1 , the mapping $u \rightarrow\{u\}$, is an order isomorphism from $\mathscr{U}(\mathfrak{U})^{\sim}$ onto $\mathscr{S}_{n}\left(\mathfrak{H}_{* 1}\right)$. Since $\mathscr{S}_{n}\left(\mathfrak{A}_{* 1}\right)$ is a complete lattice the assertion follows.

There now follows the first main result of the paper.

THEOREM 4.4. Let $\mathfrak{a}$ be a JBW*-triple and let $\mathfrak{U}_{*}$ be its predual. Then, the mapping $u \rightarrow\{u\}$, is an order isomorphism from the complete lattice $\mathscr{U}(\mathfrak{I})^{\sim}$ onto the complete lattice $\mathscr{F}_{n}\left(\mathfrak{A}_{* 1}\right)$ of norm closed faces of the unit ball $\mathfrak{U}_{* 1}$ in $\mathfrak{U}$.

Proof. By Lemma 4.1 it remains to prove that every norm-closed face $F$ of $\mathfrak{U}_{* 1}$ is norm-exposed. Let $x$ be an element of $F$ and let face $x$ be the smallest face of $\mathfrak{A}_{* 1}$ containing $x$. Then, by Corollary 4.2 ,

$$
\text { face } x \subseteq\{x\}^{\prime},=\{e(x)\},
$$

Recalling that $\{e(x)\}$, is the normal state space of the JBW-algebra $\mathfrak{A}_{2}(e(x))_{\mathrm{sa}}$ it follows from [23, Appendix 2, Lemme 6] that the norm closure face ${ }^{n}$ of face $x$ coincides with $\{e(x)\}$. When ordered by set inclusion the set $\{$ face $x: x \in F\}$, denoted by $\Gamma$, is directed, and if $x$ and $y$ are elements of $F$ such that face $x \subseteq$ face $y$ then $\{e(x)\}, \subseteq\{e(y)\}$, and $e(x) \leqslant e(y)$. Moreover, if face $x=$ face $y$, then $e(x)=e(y)$. Therefore,

$$
\{e(x) \text { : face } x \in \Gamma\}
$$

forms an increasing net in $\mathscr{U}(\mathfrak{U})$ \% However, $\left(F^{\prime}\right)$, is a norm-semi-exposed face of $\mathfrak{U}_{* 1}$ and hence, by Lemma 4.1 , of the form $\{u\}$, for some $u$ in $\mathscr{U}(\mathfrak{Q})^{\sim}$. Therefore, $F \subseteq\{u\}$, and, by Corollary 4.2 , for all elements $x$ in $F, e(x) \leqslant u$. Hence the increasing net $\{e(x)$ : face $x \in \Gamma\}$ is bounded above by $u$. Restricting to the JBW-algebra $\mathfrak{H}_{2}(u)_{\mathrm{sa}}$ and applying Lemma 4.2, the increasing net converges in the weak* topology to its supremum, an element $v$ in $\mathscr{U}(\mathfrak{l})$ with $v \leqslant u$. Notice that, for all elements $x$ in $F$, $\overline{\text { face } x}^{n}=\{e(x)\}, \subseteq\{v\}$, and it follows that $F \subseteq\{v\}$, On the other hand, if $y$ lies in $\{v\}$, the net $\left\{P_{2}(e(x))^{*} y\right.$ : face $\left.x \in \Gamma\right\}$ converges weakly to $y$. Notice that, regarded as an 
operator on $\mathfrak{U}_{2}(v)_{\mathrm{sa}}, P_{2}(e(x))$ is positive and it follows that $y$ lies in the weak closure $G^{w}$ of the set $G$ defined by

$$
G=\bigcup\left\{P_{2}(e(x))^{*}\left(\mathfrak{A}_{2}(v)_{*}^{+}\right) \cap \mathfrak{U}_{* 1}: x \in F\right\} \subseteq \bigcup\left\{\overline{\text { face } x}^{n}: x \in F\right\}=F .
$$

Since $F$ is weakly closed it follows that $\{v\}$, is contained in $F$ and hence that $\{v\}$, and $F$ coincide. Indeed, since

$$
\{u\},=\left(F^{\prime}\right),=F=\{v\},
$$

it follows that $u$ and $v$ coincide.

Notice that this result extends [16, Proposition 4], which shows that the map $u \rightarrow\{u\}$, sends the set of minimal elements of $\mathscr{U}(\mathfrak{U}) \sim$ onto the set of minimal non-empty norm closed faces of $\mathfrak{U}_{* 1}$, the set of its extreme points.

COROLLARY 4.5. Every norm-closed face of $\mathfrak{U}_{* 1}$ is norm-exposed.

The next result gives a complete description of the set of weak*-closed faces of the unit ball $\mathfrak{U}_{1}$ in the $\mathrm{JBW}^{*}$-triple $\mathfrak{A}$.

THEOREM 4.6. Let $\mathfrak{A}$ be a $\mathrm{JBW}^{*}$-triple. Then the mapping $u \rightarrow\{u\}$,' is an anti-order isomorphism from the complete lattice $\mathscr{U}(\mathfrak{U})^{\sim}$ onto the complete lattice $\mathscr{F}_{w^{*}}\left(\mathfrak{U}_{1}\right)$ of weak*-closed faces of the unit ball $\mathfrak{U}_{1}$ in $\mathfrak{U}$ and if $u$ lies in $\mathscr{U}(\mathfrak{U})$, then $\{u\}$, coincides with $u+\mathfrak{U}_{0}(u)_{1}$.

Proof. By Corollary 3.3, for each element $u$ in $\mathscr{U}(\mathfrak{A})$, the smallest element $\{u\}$, of $\mathscr{S}_{w^{*}}\left(\mathfrak{U}_{1}\right)$ containing $u$ is $u+\mathfrak{U}_{0}(u)_{1}$. Let $F$ be a weak*-semi-exposed face of $\mathfrak{U}_{1}$. Then, by Theorem 4.4 , there exists a unique element $u$ in $\mathscr{U}(\mathfrak{U})^{\sim}$ such that $F$, coincides with $\{u\}$, Therefore, by Lemma 2.1 ,

$$
F=(F)^{\prime}=\{u\}_{\prime}^{\prime} .
$$

The mapping $u \rightarrow\{u\}$, therefore sends $\mathscr{U}(A)^{\sim}$ onto $\mathscr{S}_{w}\left(\mathfrak{I}_{1}\right)$. Suppose that $u$ and $v$ are elements of $\mathscr{U}(\mathfrak{U})^{\sim}$ such that $u \leqslant v$. Then, by Lemma $3.2,\{u\}, \subseteq\{v\}$, and, by Lemma $2.1,\{v\},^{\prime} \subseteq\{u\}^{\prime}$. On the other hand, if $\{v\},{ }^{\prime} \subseteq\{u\}^{\prime}$ then

$$
\{u\},=\{u\},{ }^{\prime}, \subseteq\{v\}^{\prime},{ }^{\prime}=\{v\},
$$

and, by Theorem $4.4, u \leqslant v$. It follows that the mapping is an anti-order isomorphism. It remains to show that every non-empty weak*-closed face $F$ of $\mathfrak{U}_{1}$ is weak*-semiexposed. Notice that, since $F$, is a norm-closed face of $\mathfrak{A}_{*_{1}}$, different from $\mathfrak{A}_{* 1}$, there exists a tripotent $u$ in $\mathcal{U}$ such that $F$, coincides with $\{u\}$, and $(F)^{\prime}$ coincides with $\{u\}$, . As in the proof of Theorem 4.4, when ordered by set inclusion the set $\Gamma$ consisting of faces of $\mathfrak{U}_{1}$ of the form face $a$, where $a$ lies in $F$, is directed. By Lemma 3.5 and the above, $\{u(a)$ : face $a \in \Gamma\}$ is a decreasing net in $\mathscr{U}(\mathfrak{U})$ which, as in the proof of Lemma 4.1 , converges in the weak* topology to its infimum, a tripotent $v$. Since, for all elements $a$ in $F, v \leqslant u(a)$, it follows from Lemma 4.1 that

$$
\{v\}, \subseteq \bigcap\{\{u(a)\},: a \in F\}=\bigcap\{\{a\},: a \in F\}=F,=\{u\},
$$

and therefore $v \leqslant u$. By Lemma 3.5(i), for all elements $a$ in $F, u(a)$ lies in $F$. It follows that, for all elements $a$ in $F,\{u\}, \subseteq\{u(a)\}$, and hence that $u \leqslant u(a)$. Therefore, $u$ and $v$ coincide. Since the net $\{u(a):$ face $a \in \Gamma\}$ converges in the weak* topology to $u$ it follows that $u$ lies in $F$. If $b$ is an element of $\mathfrak{U}_{0}(u)_{1}$, by writing

$$
u=\frac{1}{2}(u+b)+\frac{1}{2}(u-b)
$$


it follows that $u+b$ lies in $F$ and hence that $u+\mathfrak{A}_{0}(u)_{1}$ is contained in $F$. Therefore,

$$
(F)^{\prime}=\{u\}_{,}^{\prime}=u+\mathfrak{U}_{0}(u)_{1} \subseteq F \subseteq(F)^{\prime},
$$

and the proof is complete.

\section{COROLLARY 4.7. Every weak*-closed face of $\mathfrak{U}_{1}$ is weak*-semi-exposed.}

Notice that for each element $a=u+b$ of $u+\mathfrak{U}_{0}(u)_{1}$ with $\|b\|<1$ we have $u(a)=u$, and in particular Theorem 4.6 then generalises [28, Theorem 6.3]. It also follows from the theorem that the maximal tripotents correspond to the minimal non-empty weak*-closed faces of $\mathfrak{U}_{1}$, namely its extreme points. This is implicit in [32] though no proof is given. In fact rather more can be said about the set of extreme points of $\mathfrak{U}_{1}$. An element $u$ in $\mathfrak{U}$ of norm one is said to be a complex extreme point of $\mathfrak{U}_{1}$ provided that for any element $a$ of $\mathfrak{U}_{1}$, the condition that the set $\{u+t a: t \in \mathbb{C},|t| \leqslant 1\}$ lies in $\mathfrak{U}_{1}$ necessarily implies that $a$ is zero. A real extreme point is similarly defined with $\mathbb{R}$ replacing $\mathbb{C}$.

\section{COROLlaRY 4.8. The following conditions are equivalent:}

(i) $u$ is a maximal element of $\mathscr{U}(\mathfrak{U})$;

(ii) $\mathfrak{U}_{0}(u)=\{0\}$;

(iii) $u$ is a real extreme point;

(iv) $u$ is a complex extreme point;

(v) $u$ is an extreme point.

Proof. (i) $\Rightarrow$ (ii) Suppose that $\mathfrak{A}_{0}(u) \neq\{0\}$. Since $\mathscr{U}\left(\mathfrak{\Re}_{0}(u)\right)$ contains a non-zero element $v$ it follows that $u \leqslant u+v$, contradicting the maximality of $u$.

(ii) $\Rightarrow$ (i) If $u$ is a tripotent such that $\mathfrak{A}_{0}(u)=\{0\}$ and $u \leqslant v$. Then $v-u$ lies in $\mathfrak{U}_{0}(u)$, and $v=u$ as required.

(ii) $\Rightarrow$ (iii) Let $u$ be a maximal tripotent and let $a$ be an element of $\mathfrak{U}_{1}$ such that for all real numbers $t$ in $[-1,1], u+t a$ lies in $\mathfrak{X}_{1}$. By [16, Corollary 1.2(b)] $\left(P_{2}(u)+P_{0}(u)\right)(u+t a)$ is an element of $\mathfrak{U}_{1}$, from which it follows that $u+t P_{2}(u) a$ is an element of $\mathfrak{U}_{1}$. Therefore, $u+t P_{2}(u) a$ is an element of the unit ball $\mathfrak{A}_{2}(u)_{1}$ in the Jordan $\mathrm{W}^{*}$-algebra $\mathfrak{U}_{2}(u)$. The same is true of $u-t P_{2}(u) a$ and therefore, since

$$
u=\frac{1}{2}\left(u+t P_{2}(u) a\right)+\frac{1}{2}\left(u-t P_{2}(u) a\right),
$$

Lemma 2.2 shows that $P_{2}(u) a=0$. Therefore, $P_{1}(u) a=a$ and $u+t P_{1}(u) a$ is an element of $\mathfrak{U}_{1}$. Moreover, $P_{2}(u)\left(u+t P_{1}(u) a\right)=u$ and since the proof of [16, Lemma 1.6] applies to any non-zero element of $\mathfrak{U}_{1}$, it follows that $a=P_{1}(u) a=0$.

(iii) $\Rightarrow$ (iv) This follows from the definition.

(iv) $\Rightarrow$ (ii) This is given in [27, Proposition 3.5].

(v) $\Rightarrow$ (i) This follows immediately from Theorem 4.5.

\section{Applications}

The results of $\$ 4$ describe the facial structure of the unit balls in a weak*-closed $\mathrm{J}^{*}$-algebra and in its predual. In particular they apply to $\mathrm{W}^{*}$-algebras. In these examples the set $\mathscr{U}(\mathfrak{U})$ of tripotents coincides with the set of partial isometries. Notice that if $u$ and $v$ are elements of $\mathscr{U}(\mathfrak{U})$ such that $u \leqslant v$, then their initial and final 
projections satisfy $u u^{*} \leqslant v v^{*}$ and $u^{*} u \leqslant v^{*} v$. Moreover, $u$ and $v$ are orthogonal if and only if one of the following three equivalent conditions hold:

$$
\begin{gathered}
u v^{*} u=0 \\
u v^{*}=v u^{*}=0 \\
u u^{*} \perp v v^{*}, \quad u^{*} u \perp v^{*} v .
\end{gathered}
$$

When, as before, $\mathscr{U}(\mathfrak{A})^{\sim}$ denotes the set of partial isometries in $\mathfrak{U}$ with a largest element adjoined its properties can be summarised in the following manner.

THEOREM 5.1. Let $\mathfrak{A}$ be a weak*-closed $\mathrm{J}^{*}$-algebra and let $\mathscr{U}(\mathfrak{U})^{\sim}$ be the set of partial isometries in $\mathfrak{U}$ with an element $\omega$ adjoined. Then, with respect to the partial ordering defined for two partial isometries $u$ and $v$ in $\mathfrak{A}$ by the conditions that $u \leqslant v$ if and only if $u v^{*} u=u$ and, for all elements $u$ in $\mathscr{U}(\mathfrak{U})^{\sim}, u \leqslant \omega, \mathscr{U}(\mathfrak{U})^{\sim}$ forms a complete lattice.

In the special case when $\mathfrak{A}$ is a $\mathrm{W}^{*}$-algebra a little more can be said.

COROLlaRY 5.2. Let $A$ be $a \mathrm{~W}^{*}$-algebra and let $\mathscr{U}(\mathfrak{U})^{\sim}$ be the complete lattice of partial isometries in $\mathfrak{U}$ with a largest element adjoined. Then the set $\mathscr{P}(\mathfrak{U})$ of self-adjoint idempotents in $\mathfrak{U}$ is a principal ideal in $\mathscr{U}(\mathfrak{U})^{\sim}$ generated by the unit $e$ in $A$ (that is, $\left.\mathscr{P}(\mathfrak{U})=\left\{u \in \mathscr{U}(\mathfrak{U})^{\sim}: u \leqslant e\right\}\right)$.

Proof. It is clearly enough to show that if $u$ is an element of $\mathscr{U}(\mathfrak{U})$ and $p$ is an element of $\mathscr{P}(\mathfrak{U})$ such that $u \leqslant p$ then $u$ lies in $\mathscr{P}(\mathfrak{U})$. If this is the case then $\mathfrak{A}_{2}(p)$ is the sub-W*-algebra of $\mathfrak{U}$ consisting of elements $a$ of $\mathfrak{U}$ such that $a=$ pap. By Lemma 2.4 it follows that $u$ is a self-adjoint idempotent in $\mathfrak{U}_{2}(p)$ and hence an element of $\mathscr{P}(\mathfrak{U})$ as required. result.

When applied to weak*-closed $\mathrm{J}^{*}$-algebras Theorem 4.4 yields the following

THEOREM 5.3. Let $\mathfrak{U}$ be a weak*-closed $\mathrm{J}^{*}$-algebra. Then every norm-closed face $F$ of the unit ball $\mathfrak{U}_{* 1}$ in the predual $\mathfrak{U}_{*}$ of $\mathfrak{U}$ not equal to $\mathfrak{U}_{* 1}$ is of the form

$$
\{u\},=\left\{x: x \in \mathfrak{X}_{* 1}: x(u)=1\right\}
$$

for some unique element $u$ of the set $\mathscr{U}(\mathfrak{U})$ of partial isometries in $\mathfrak{U}$. Alternatively, $F$ consists of the set of all elements $x$ in $\mathfrak{U}_{* 1}$ such that, for all elements $a$ in $\mathfrak{U}$,

$$
\overline{x\left(u u^{*} a u^{*} u\right)}=x\left(u a^{*} u\right)
$$

and

$$
x\left(u a^{*} u u^{*} a u^{*} u\right) \geqslant 0 .
$$

Moreover, the mapping $u \rightarrow\{u\}$, is an order isomorphism from the complete lattice $\mathscr{U}(\mathfrak{A})^{\sim}$ onto the complete lattice $\mathscr{F}_{n}\left(\mathfrak{A}_{* 1}\right)$ of norm closed faces of $\mathfrak{U}_{* 1}$.

Proof. The result follows from Theorem 4.4 and the observation that $F$ can be regarded as the normal state space of the Jordan $W^{*}$-algebra $\mathfrak{A}_{2}(u)$.

The final result extends [20, Theorem 11], where the extreme points of the unit ball in a $\mathbf{J}^{*}$-algebra are described in terms of partial isometries. When applied to $\mathrm{W}^{*}$ - 
algebras it extends [29, Proposition 1.6.5]. Its proof follows immediately from Theorem 4.6.

THEOREM 5.3. Let $\mathfrak{A}$ be a weak*-closed $\mathrm{J}^{*}$-algebra. Then every non-empty weak* closed face of the unit ball $\mathfrak{U}_{1}$ in $\mathfrak{U}$ is of the form

$$
\{u\}_{,}^{\prime}=u+\left(1-u u^{*}\right) \mathfrak{U}\left(1-u^{*} u\right)_{1}
$$

for some unique element $u$ of the set $\mathscr{U}(\mathfrak{U})$ of partial isometries in $\mathfrak{U}$. Moreover, the mapping $u \rightarrow\{u\}, '$ is an anti-order isomorphism from the complete lattice $\mathscr{U}(\mathfrak{U})$ 'onto the complete lattice $\mathscr{F}_{w^{*}}\left(\mathfrak{U}_{1}\right)$ of weak*-closed faces of $\mathfrak{U}_{1}$.

\section{References}

1. E. M. AlfSEn and F. W. Shultz, 'On non-commutative spectral theory and Jordan algebras', Proc. London Math. Soc. 38 (1979) 335-344.

2. E. M. Alfsen, F. W. ShultZ and E. STORMer, 'A Gelfand-Naimark theorem Jordan algebras', $A d v$. in Math. 28 (1978) 11-56.

3. L. Asimow and A. J. Elus, Convexity theory and its applications in functional analysis (Academic Press, London, 1980).

4. T. Barton and R. M. Timoney, 'On biduals, preduals and ideals of JB*-triples', Math. Scand. to appear.

5. S. DinEEN, 'The second dual of a JB*-triple system', Complex analysis, functional analysis and approximation theory, North-Holland Mathematical Studies 125 (North-Holland, Amsterdam, 1986) 67-69.

6. C. M. EDWARDS, 'Ideal theory in JB-algebras', J. London Math. Soc. (2) 16 (1977) 507-513.

7. C. M. Edwards, 'On the facial structure of a JB-algebra', J. London Math. Soc. (2) 19 (1979) 335-344.

8. C. M. Edwards, 'On Jordan W*-algebras', Bull. Soc. Math. 104 (1980) 393-403.

9. C. M. EdWARDS and G. T. RürtimanN, 'Isometries of GL-spaces', J. London Math. Soc. (2) 31 (1985) $125-130$.

10. C. M. Edwards and G. T. RürtimanN, "On the facial structure of the unit balls in a GL-space and its dual', Math. Proc. Cambridge Philos. Soc. 98 (1985) 305-322.

11. C. M. EdWARDS and G. T. RüTtimanN, 'On the facial structure of the unit balls in a GM-space and its dual', Math. Z. 193 (1986) 597-611.

12. Y. Friedman and B. Russo, 'Contractive projections on operator triple systems', Math. Scand. 52 (1983) 279-311.

13. Y. Friedman and B. Russo, 'Function representation of commutative operator triple systems', J. London Math. Soc. (2) 27 (1983) 513-524.

14. Y. Friedman and B. Russo, 'Conditional expectation without order', Pacific J. Math. 115 (1984) $351-360$.

15. Y. Friedman and B. Russo, 'Solution of the contractive projection problem', J. Funct. Anal. 60 (1985) 56-79.

16. Y. Friedman and B. Russo, 'Structure of the predual of a JBW*-triple', J. Reine Angew. Math. 356 (1985) 67-89.

17. Y. Friedman and B. Russo, 'The Gelfand-Naimark theorem for JB'-triples, Duke Math. J. 53 (1986) 139-148.

18. Y. Friedman and B. Russo, 'Conditional expectation and bicontractive projections on Jordan C*algebras and their generalisations', Math. Z. 194 (1987) 227-236.

19. H. HANCHE-OlSEN and E. STORMER, Jordan operator algebras (Pitman, London, 1984).

20. L. A. HARris, 'Bounded symmetric homogeneous domains in infinite-dimensional spaces', Lecture Notes in Mathematics 364 (Springer, Berlin, 1974) 13-40.

21. L. A. Harris, 'A generalization of C*-algebras', Proc. London Math. Soc. (3) 42 (1981) 331-361.

22. G. HoRN, 'Klassifikation der JBW*-tripeln vom Typ I', Dissertation, Tübingen 1984.

23. В. Іосним, 'Cônes autopolaires et algèbres de Jordan', Lecture Notes in Mathematics 1049 (Springer, Berlin, 1984).

24. W. KaUP, 'Algebraic characterizations of symmetric complex Banach manifolds', Math. Ann. 228 (1977) 39-64.

25. W. KAUP, 'A Riemann mapping theorem for bounded symmetric domains in complex Banach spaces', Math. Z. 1983 (1983) 502-529.

26. W. KAUP, 'Contractive projections on Jordan $\mathrm{C}^{*}$-algebras and generalizations', Math. Scand. 54 (1984) 95-100. 
27. W. KaUP and H. UpMEIER, 'Jordan algebras and symmetric Siegel domains in Banach spaces', Math. Z. 157 (1977) 179-200.

28. O. Loos, Bounded symmetric domains and Jordan pairs (University of California, Irvine, 1977).

29. S. SAKAI, C*-algebras and $\mathrm{W}^{*}$-algebras (Springer, Berlin, 1971).

30. F. W. SHultz, 'On normed Jordan algebras which are Banach dual spaces', J. Funct. Anal. 31 (1979) $360-376$.

31. H. UPMEIER, Symmetric Banach manifolds and Jordan $\mathrm{C}^{*}$-algebras (North-Holland, Amsterdam, 1985).

32. H. UPMEIER, Jordan algebras in analysis, operator theory, and quantum mechanics (American Mathematical Society, Providence, 1986).

33. J. D. M. WRIGHT, 'Jordan C*-algebras', Michigan Math. J. 24 (1977) 291-302.

34. M. A. Youngson, 'A Vidav theorem for Banach Jordan algebras', Math. Proc. Cambridge Philos. Soc. 84 (1978) 263-272.

The Queen's College Oxford OX1 4AW
Institute of Mathematical Statistics

University of Berne

Switzerland 